\title{
INTERFERON ALPHA2B INDUCED DOPAMINE CHANGES IN CUTANEOUS MELANOMA PATIENTS
}

\author{
Corina-Daniela Ene ${ }^{1}$, Ilinca Nicolae ${ }^{2}$ \\ 1 "Carol Davila" Clinical Hospital of Nephrology, Bucharest, Romania \\ ${ }^{2}$ Research Department in Dermatology, "Victor Babes" Clinical Hospital of Tropical and Infectious \\ Diseases, Bucharest, Romania
}

\begin{abstract}
Objective. The authors interest was focused on interferon impact on dopamine status and on the relation between negative emotional state and dopamine in melanoma patients.

Methods. 60 patients diagnosed with malignant melanoma in 1st or 2nd clinical stage were included in the first 56 days after surgical removal of the tumor in an observational prospective study. The patients were divided in 2 groups: group $A$ that included 30 cases treated with $10 \mathrm{MU}$ interferon alpha2b/mp three times a week for one year and group $\mathrm{B}$ that included 30 cases with no adjuvant treatment. Urinary dopamine (ELISA) was evaluated before treatment with interferon alpha2b, after 1, 6, 12 months of treatment and after 6 months from the end of the treatment.

Neuropsychiatric disorders were grouped according to their frequency in melanoma patients.

Results. Neuropsychiatric disorders associated with the treatment with interferon were: irritability, asthenia and fatigability, sleep disorders, anxiety, cognitive disorders, somatic symptoms. The treatment with interferon altered dopamine metabolism. Dopamine returned to the pretherapeutical values at six months after interferon was stopped. Patients with low levels of urinary dopamine had a high, statistically significant risk of developing depression during interferon treatment $(\mathrm{OR}=2.647, \mathrm{IC}=2.186-3.014, \mathrm{p}=0.0216)$.

Conclusions. Low dopamine might have a major role in the development of depression secondary to interferon treatment.
\end{abstract}

Keywords: dopamine, interferon alpha2b, melanoma, neuropsychiatric disorders

\section{INTRODUCTION}

Radical surgery with safety margins, histologically confirmed, offers the best chance of curing early stages of malignant melanoma. Patients with 2A, 2B and 3B stages of melanoma according AJCC (American Joint Committee on Cancer), with high risk of recurrence, receive adjuvant systemic therapy. Multiple studies (1-6) evaluated the role of adjuvant therapy with interferon alpha $2 b$ (IFN) in malignant melanoma. Most patients who received IFN presented liver, hematologic, neuropsychiatric and constitutional toxicity. The frequency of neuropsychiatric disorders was influenced by the administration of IFN, dose and time of administration, mental status of patients. A variety of neuropsychiatric disorders associated with
IFN treatment was described in melanoma patients: depressive symptoms, anxiety, cognitive, neurovegetative, somatic symptoms) (1-7).

Ethiopathogenic hypothesis of depressive syndrome were based on multifactoriality in relation with neuro-immune-endocrine triade (7-14). Neurobiochemical vulnerability was demonstrated by serotoninergic, noradrenergic, dopaminergic, acetilcholinergic, GABA-ergic and peptidergic implications. Immunological disorders that appear in depression were correlated with cytokines overexpression (TNFa, IL-1, IL-6), signal pathways altering (NF-kB, MAPK), overloading of some mediators of inflammation (COX2, RNS/ROS). Stress was associated with hypo-thalami-adrenal ax overexpression, with hyperactivity of sympathomimetic

Author for correspondence:

Ilinca Nicolae, "Victor Babes" Clinical Hospital of Tropical and Infectious Diseases, Bucharest, Romania

E-mail: drnicolaei@yahoo.ro 
system and of adrenergic components of adrenal gland.

The adjustment of hypothalamus-pituitary-adrenal axis activity can be achieved by monoamine intervention. BEISA dysfunction (brain, endocrine, immune and sympathoadrenal axes) generates imbalances in various signaling and transduction systems. Recent data referring to the etiology of depressive disorders tend to integrate conceptual monoaminergic, neuroendocrine and immunologic systems (14).

Interferon proved to be an inductor for synthesis of proinflamatory cytokines (IL-1, IL-2, IL-6, TNF alpha) that are involved in neurotransmitters metabolism. Depression development during the treatment with interferon alpha2b was associated with altered dopaminergic and serotoninergic transmission $(7,8)$. Serotonin status in human body could be influenced by interferon through two mechanisms:

- activation of indolamine-2,3-deoxygenase, followed by decreased concentration of triptofan and reduced synthesis of serotonin $(7,9)$;

- stimulation of MAPK (p38) associated with increased active transport mechanisms and high serotonin reuptake (10).

Interferon could influence dopamine levels through multiple mechanisms:

- decrease of tetrahydrobiopterin (BH4) levels, stimulation of nitric oxid and interleukin 6 synthesis and inactivation of tyrosinhydroxylase;

- activation of MAPK/MEK, overexpression of dopamine transport and increase of dopamine reuptake;

- activation of indolamine-2,3- deoxygenase, decrease of triptofan levels, stimulation of kynurenic acid synthesis, inhibition of glutamate release and decrease of dopamine synthesis.

The present study aimed to evaluate interferon impact on dopamine status and a possible relation between neuropsychiatric disorders and dopamine variation in melanoma patients. Such approaches could lead to identification of useful items in clarifying the depression pathophysiology and to establish possible preventive pharmacological interventions in this pathology.

\section{METHODS}

The present study was based on a five years prospective analysis and was developed in Center of Research in Dermatology from Clinical Hospital Victor Babes, Bucharest, Romania. The study was approved by the Ethics Committee of the Hospital. All the patients were diagnosed with melanoma stage I-II by histopathological exam and included in the study in the first 56 days after surgical removal of the tumor.

Exclusion criteria:

- pregnancy;

- alcohol, drug consume;

- hormonal therapy, antidepressant, antioxidant, immunomodulatory therapy dopamine receptors blockers, mono-amino-oxidase inhibitors;

- treated malignant melanoma;

- associated disorders: neurological, psychiatric, digestive, endocrinological, cardiovascular, hepatic, renal, pulmonary, metabolic, autoimmune, infections/inflammation, others neoplastic processes;

- high values for some biochemical and hematological markers that can give false positive reactions (high levels of triglycerides, conjugated bilirubin, unconjugated bilirubin, serum hemoglobin over $500 \mathrm{mg} / \mathrm{dl}$ );

- refuse to participate to the study.

All the participants in the study signed the informed consent according to the Declaration of Helsinki from 1964.

60 patients with melanoma stages I-II were selected and dived in two groups:

- group A that included 30 patients with malignant melanoma treated with interferon alpha2b 10MU IFNxmp ${ }^{-1}$, subcutaneous, three times a week, for one year. Group A included 20 women and 10 men with age average $52.9 \pm 13.4$ years.

- group B that included 30 patients with malignant melanoma without treatment with interferon alpha2b. The decision for no treatment was taken by the oncologist according to patient desire. Group B included 20 women and 10 men with age average $50.5 \pm 14.0$ years.

The laboratory assessments were made in all the patients at five moments:

- moment 1 - eight weeks after surgical removal of the tumor, respectively, before the treatment start;

- moment 2 - one month of treatment, respectively, three months after surgical removal of the tumor;

- moment 3 - six months of treatment, respectively, eight months after surgical removal of the tumor;

- moment 4 - twelve months of treatment, respectively, 14 months after surgical removal of the tumor; 
- moment 5 - six months after the treatment, respectively, 20 months after surgical removal of the tumor;

In all the patients included in the study were assessed: hematological parameters (Pentra C 60 analyzer), biochemical tests (HumaStar300), urinary dopamine (ELISA) (Table 1).

TABLE 1. Demographic and biological characteristics in patients with malignant melanoma

\begin{tabular}{|l|c|c|}
\hline Characteristics & $\begin{array}{c}\text { Melanoma -IFN } \\
(\mathrm{N}=30)\end{array}$ & $\begin{array}{c}\text { Melanoma }- \text { nonIFN } \\
(\mathrm{N}=30)\end{array}$ \\
\hline Sex: men/women & $1: 2$ & $1: 2$ \\
\hline Age (years) & $52.9 \pm 13.4$ & $50.5 \pm 14.0$ \\
\hline BMI (kg/m $\left.{ }^{2}\right)$ & $23.8 \pm 1.9$ & $24.1 \pm 1.6$ \\
\hline Hemoglobin (g/dl) & $12.6 \pm 1.3$ & $12.9 \pm 2.1$ \\
\hline Leucocytes (cel/mmc) & $5400 \pm 1100$ & $5650 \pm 935$ \\
\hline Platelets x10 $(/ \mathrm{mmc})$ & $237 \pm 31$ & $286 \pm 93$ \\
\hline Glucose $(\mathrm{mg} / \mathrm{dl})$ & $88.3 \pm 16.2$ & $91.5 \pm 12.4$ \\
\hline ALT (U/L) & $19.4 \pm 6.2$ & $21.8 \pm 8.3$ \\
\hline AST (U/L) & $20.4 \pm 8,3$ & $19.2 \pm 5.7$ \\
\hline Cholesterol (mg/dl) & $150 \pm 30.8$ & $178 \pm 24.5$ \\
\hline HDL-cholesterol (mg/dl) & $47.4 \pm 8.1$ & $51.9 \pm 9.5$ \\
\hline Triglycerides (mg/dl) & $81.4 \pm 22.9$ & $77.2 \pm 19.4$ \\
\hline Urea $(\mathrm{mg} / \mathrm{dl})$ & $26.1 \pm 9.3$ & $31.7 \pm 12.3$ \\
\hline Creatinine $(\mathrm{mg} / \mathrm{dl})$ & $0.81 \pm 0.14$ & $0.78 \pm 0.09$ \\
\hline Dopamine $(\mathrm{pg} / \mathrm{ml})$ & $47.1 \pm 13.3$ & $51.4 \pm 9.6$ \\
\hline Dopamine(ug//24h) & $171.3 \pm 42.8$ & $178.7 \pm 57.1$ \\
\hline
\end{tabular}

Neuropsychiatric adverse reactions were grouped by frequency: very frequent (affects 1 from 10 patients), frequent (affects 1 to 10 from 100 patients), less frequent (affects 1 to 10 from 1,000 patients) and rare (affects 1 to 10 from 10,000 patients).

Statistical analysis. All data were statistically evaluated using SPSS, a specialized soft for statisti- cal analysis. The results were presented as mean \pm standard deviation. $t$ test or ANOVA tests were used to compare two groups of variables. Odds Ratio (OR) was used for testing the dependence between two factors. We also evaluated the survival rate in melanoma patients treated with interferon alfa2b by dopamine levels using Kaplan-Meyer curves.

\section{RESULTS AND DISCUSSIONS}

Results. Neuropsychiatric effects of interferon were evaluated in two homogenous groups for biological profile and demographical characteristics: 30 patients with melanoma treated with interferon alpha2b and 30 patients with melanoma without adjuvant treatment.

The systemic therapy with IFN in melanoma patients was associated with neuropsychiatric disorders, evaluated as follows: 1) very frequent: asthenia, fatigue, irritability, sleep disorders; 2) frequent: fear, lack of concentration, indecisiveness, irritative urinary symptoms; 3) less frequent: memory disturbance, muscle or joint pain, gastrointestinal symptoms. Psychiatric disorders were observed in over $75 \%$ patients during the first month of treatment with IFN, other disorders being registered in the next six months of treatment. Vary rare depressive reactions were noticed in patients with melanoma without treatment. In conclusion, neuropsychiatric alterations were secondary to interferon alpha2b (Fig. 1).

Interferon influence on dopamine in melanoma patients versus melanoma non-IFN patients was analyzed by sex and age (Table 2), and also, for the

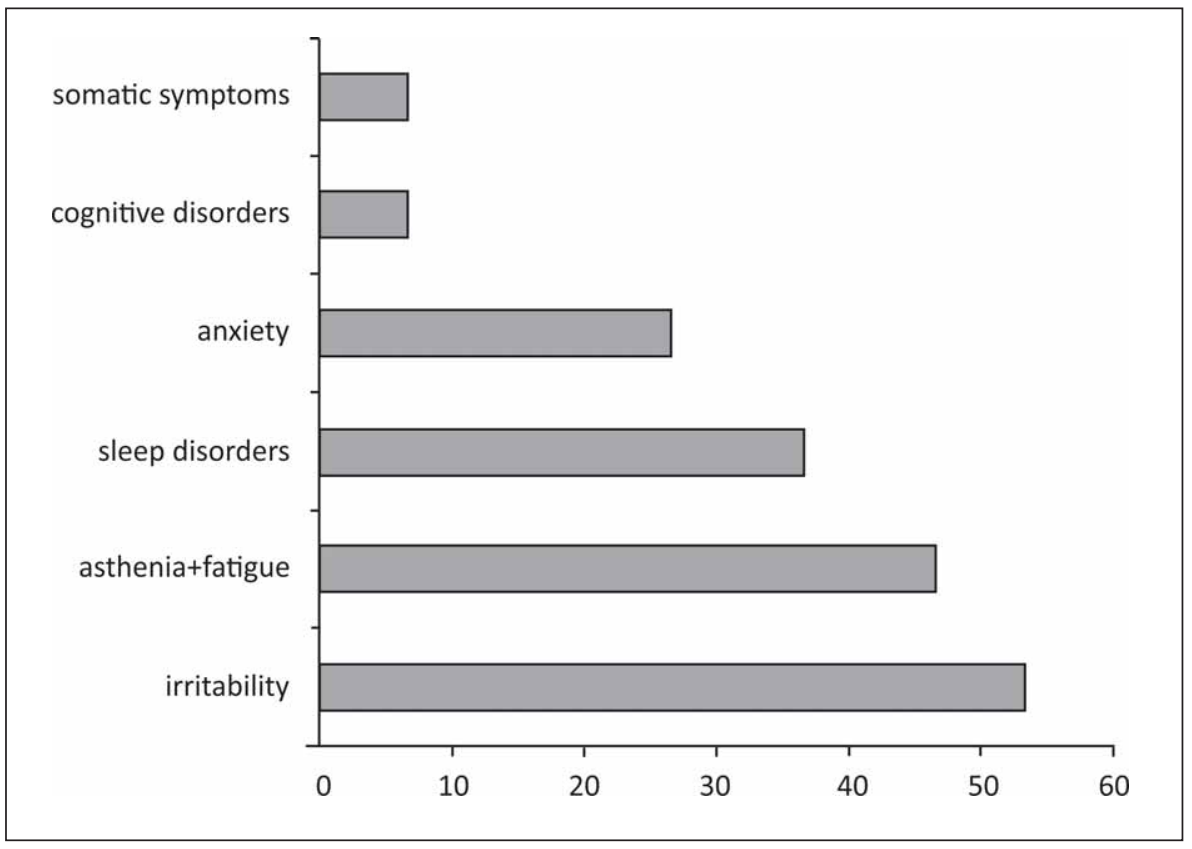

FIGURE 1. Interferon associated neuropsychiatric effects in melanoma patients 
whole group (Fig. 2). It should be noted that the production of dopamine is intermittent, and the determination of dopamine in urine is more appropriate than its determination in plasma (Table 1).

In melanoma women in premenopausal, during the treatment with interferon alpha2b, dopamine decreased compared to the moment $1(186.00 / 50.28$ $\mathrm{ug} / 24 \mathrm{~h})$ with $12.5 \%(\mathrm{p}=0.389, \mathrm{IC}=95 \%)$, after one month, with $27.36 \%(p=0.017, \mathrm{IC}=95 \%)$ after six months, with $21.71 \%(\mathrm{p}=0.047, \mathrm{IC}=95 \%)$ after 12 months of treatment. At moment 5 (six months after the treatment was stopped), dopamine returned to pre-treatment values. Dopamine levels did not vary in premenopausal melanoma women untreated with interferon alpha2b (Table 2).

TABLE 2. Dopamine variation (ug/24h) in melanoma patients during treatment with interferon alfa $2 b$

\begin{tabular}{|l|c|c|c|}
\hline & $\begin{array}{c}\text { Premenopausal } \\
\text { women }\end{array}$ & $\begin{array}{c}\text { Postmenopausal } \\
\text { women }\end{array}$ & Men \\
\hline IFN $(\mathrm{N}=30)$ & \\
\hline 1 & $195.30 \pm 49.99$ & $159.80 \pm 62.94$ & $184.80 \pm 47.88$ \\
\hline 2 & $170.90 \pm 46.94$ & $170.90 \pm 79.92$ & $180.80 \pm 38.12$ \\
\hline 3 & $158.60 \pm 42.06$ & $154.60 \pm 58.83$ & $165.50 \pm 42.92$ \\
\hline 4 & $195.80 \pm 44.21$ & $145.20 \pm 55.89$ & $165.60 \pm 42.17$ \\
\hline 5 & $194.90 \pm 49.68$ & $159.90 \pm 62.71$ & $184.80 \pm 47.88$ \\
\hline nonIFN $(\mathrm{N}=30)$ & & \\
\hline 1 & $186.00 \pm 50.28$ & $168.40 \pm 45.76$ & $160.90 \pm 38.86$ \\
\hline 2 & $162.70 \pm 49.58$ & $* 116.50 \pm 38.42$ & $* 125.00 \pm 28.89$ \\
\hline 3 & $* 135.10 \pm 60.25$ & $150.40 \pm 64.47$ & $* 123.60 \pm 38.80$ \\
\hline 4 & $* 145.80 \pm 29.74$ & $159.30 \pm 30.27$ & $143.40 \pm 38.03$ \\
\hline 5 & $189.90 \pm 65.51$ & $168.60 \pm 45.38$ & $160.90 \pm 38.73$ \\
\hline
\end{tabular}

$1,2,3,4,5$ - moments of evaluation anterior defined

$\left({ }^{*} \mathrm{p}<0.05\right.$ versus moment $\left.1, \mathrm{IC}=95 \%\right)$

In postmenopausal melanoma women, dopamine dynamics during IFN treatment was similar.
Compared to moment 1, (166.40/45.76ug/24h), dopamine decreased with $30.84 \%$ after one month $(\mathrm{p}=0.002, \mathrm{IC}=95 \%)$, with $11.47 \%$ after six months $(\mathrm{p}=0.073, \quad \mathrm{IC}=95 \%), \quad$ with $5.22 \% \quad(\mathrm{p}=0.097$, $\mathrm{IC}=95 \%$ ) after twelve months of treatment with interferon alpha2b. After the treatment was stopped, dopamine increased slowly and it had the same pretherapeutically values at six months after the treatment (Table 2). Dopamine levels did not vary in postmenopausal melanoma women without treatment with interferon alpha2b (Table 2).

In melanoma men treated with interferon alpha2b, dopamine decreased significantly compared to moment $1(160.90 / 38.86 \mathrm{ug} / 24 \mathrm{~h})$, with $21.92 \%$ after one month ( $\mathrm{p}=0.007, \mathrm{IC}=95 \%$ ), with $22.82 \%$ after six months ( $\mathrm{p}=0.003, \mathrm{IC}=95 \%$ ), with $10.37 \%$ after twelve months of treatment $(p=0.103$, $\mathrm{IC}=95 \%$ ). Dopamine levels normalized at six months after the adjuvant treatment was stopped (Table 2).

The statistical analysis showed significantly increased levels of dopamine in group A compared with group B (Fig. 2), variation that confirmed the results presented above.

The role of dopamine in development of depression in melanoma patients treated with interferon alpha2b was evaluated by odds ratio and $\mathrm{p}$-value. The values for $\mathrm{OR}=2.647$, IC $=2.186-3.014$, $\mathrm{p}=0.021$ showed that patients with low levels of dopamine have a higher risk, statistical significantly, for developing neuropsychiatric disorders during the treatment with interferon.

The authors also analyzed the influence of dopamine on the relapse rate in melanoma patients. The survival rate relapse free (SRR) in melanoma patients treated with interferon alpha2b by dopamine

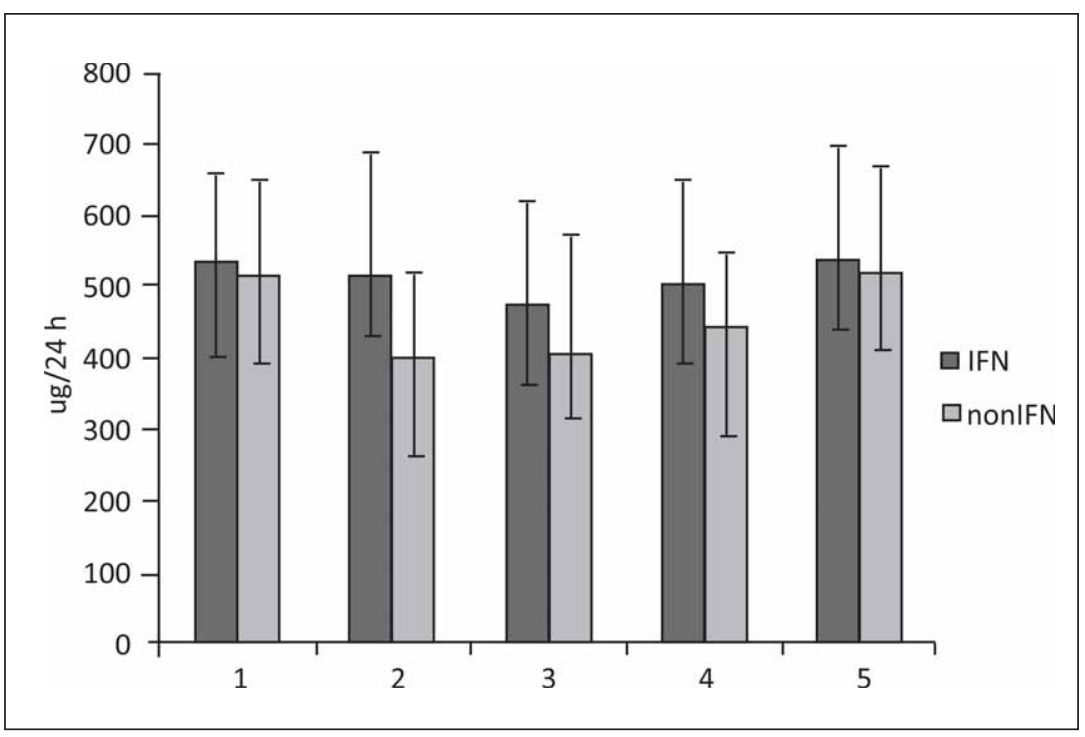

FIGURE 2. Dopamine dynamics in melanoma patients treated with IFN versus nonIFN melanoma patients 
levels indicated a SRR of $18.47 \pm 0.73$ months for urinary dopamine under $171 \mathrm{ug} / 24 \mathrm{~h}$ and $19.28 \pm 2.71$ months for urinary dopamine over $171 \mathrm{ug} / 24 \mathrm{~h}$ in group A. In group B, SRR was $19.28 \pm 1.40$ months for urinary dopamine under $171 \mathrm{ug} / 24 \mathrm{~h}$ and $20.55 \pm 1.14$ months for urinary dopamine over 171 $\mathrm{ug} / 24 \mathrm{~h}$. The survival rate was higher, but without statistical significance, in melanoma patients with increased levels of dopamine compared with melanoma patients with decreased levels of dopamine ( $\mathrm{p}>0,05)$ (Fig. 3).

Discussions. Systemic therapy with interferon alpha2b determined early appearance of neuropsychiatric disorders, with a significant frequency (Fig. 1). This observation is strengthened by the finding that the incidence of depression in patients with non-IFN melanoma is very rare.

IFN therapy induced alteration of dopamine metabolism (Table 2), resulted in a reduction of dopamine up to $40 \%$ during the treatment with IFN (Fig. 2 ). After the treatment was stopped, dopamine returned to the values comparable with the initial ones. Therefore, reduction of dopamine associated with IFN treatment was reversible and non-cumulative.

Decreased dopamine in patients with melanoma is a risk factor for depressive symptoms secondary to IFN therapy $(\mathrm{OR}=2.647, \mathrm{IC}=2.186-3.014$, $\mathrm{p}=0.0216$ ). This association between depressive episodes and impaired status of dopamine may have a role in the etiology of neuropsychiatric disorders.

In literature no categorical statements about the relationship between dopamine and neuropsychiat- ric disorders secondary to IFN therapy were observed. Recent data tend to give the dopaminergic hypothesis an important role in the etiology of depressive disorders (15-19). Blocking the reuptake of dopamine (nomifensine) or modulation of dopamine receptors (pramipexile) showed reduced depressive symptoms (17). In cerebrospinal fluid and plasma from the jugular vein, reduced amounts of dopamine metabolites were found in depression, suggesting the decrease of dopamine turnover (18). Reduced expression of dopamine transporter in striatum was associated with reduced dopamine reuptake and, consequently, reduced dopaminergic neurotransmission (19). IFN stimulates the indolamine-2,3-dioxygenase activity and decreases the protective action of factors influencing oxidative and apoptotic mechanisms, with altered expression of growth factor BDNF (brain-derived neurotrophic factor) (20). There are a number of mechanisms by which IFN may alter dopamine metabolism: activation of indolamine-2,3-dioxygenase (13), inactivation of tyrosinhidroxilases (11), alteration of some signaling pathways (12).

To investigate the relationship between the severity of neuropsychiatric events and profile of dopamine, the authors recommend the dosage of dopamine in urine, because the test is more appropriate than testing plasma dopamine. This test would be able to identify patients prone to develop depression secondary to the treatment with IFN. Identifying patients with depression in the early stage requires attention and close collaboration of the therapeutic team to initiate a specific treatment.

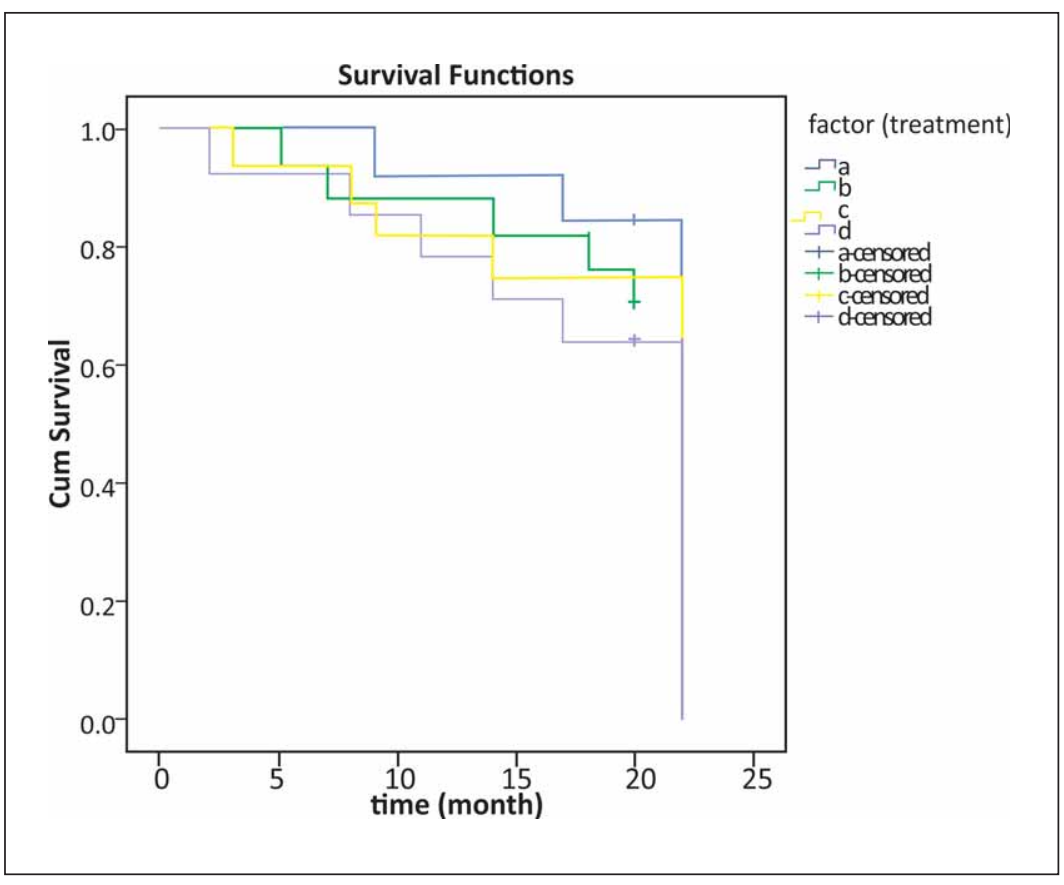

FIGURE 3. Kaplan-Meier relapse-free survival curves in group $A$ and group $B$ relative to urinary levels of dopamine (a - IFN group, urinary dopamine $<171$ ug/24 h, b - IFN group, urinary dopamine $>171 \mathrm{ug} / 24 \mathrm{~h}, \mathrm{c}-$ non INF group, urinary dopamine $<171 \mathrm{ug} / 24 \mathrm{~h}, d-$ nonINF group, urinary dopamine $>171 \mathrm{ug} / 24 \mathrm{~h}$ ) 
The analysis of relapse-free survival curves in patients with malignant melanoma by dopamine status showed differences between IFN and nonIFN groups with respect to survival without relapse. Thus, in group A, in patients with malignant melanoma with elevated urinary dopamine was estimated a prolonged disease-free survival rate than in patients with malignant melanoma with low urinary dopamine. The same correlation between dopamine levels and relapse-free survival was achieved in group B. For the same urinary dopamine status in patients in IFN group, there was estimated lower relapse-free survival compared with nonIFN group. As a result, it can be accepted that the reduction of dopamine could be a negative predictive factor in assessing the response to interferon alpha2b.

\section{REFERENCES}

1. Hauschild A. Adjuvant interferon alfa for melanoma: new evidencebased treatment recommendations?. Curr Oncol 2009; 16(3):3-6

2. Hauschild A., Schadendorf D., Garbe C., Ugurel S., Kähler K.C. New promises in the adjuvant, and palliative treatment of melanoma. Cancer Treat Res 2007; 135:277-92

3. Hauschild A., Gogas H., Tarhini A., et al. Practical guidelines for the management of interferon-alpha- $2 b$ side effects in patients receiving adjuvant treatment for melanoma:expert opinion. Cancer 2008; 112:982-94

4. Dummer R., Hauschild A., Jost L. On behalf of the esmo Guidelines Working Group. Cutaneous malignant melanoma: esmo clinical recommendations for diagnosis, treatment and follow-up. Ann Oncol 2008; 19(2):ii86-8

5. Pectasides D., Dafni U., Bafaloukos D., et al. Randomized phase III study of 1 month versus 1 year of adjuvant high-dose interferon alfa-2b in patients with resected high-risk melanoma. J Clin Oncol 2009; 27:939-44

6. Eggermont A.M., Suciu S., Testori A., et al. Long-term results of the randomized phase III trial EORTC 18991 of adjuvant therapy with pegylated interferon alfa-2b versus observation in resected stage III melanoma. J Clin Oncol 2012; 30(31):3810-18

7. Capuron L., Gumnick J.F. Neurobehavioral effects of interferon alpha in cancer patients. Neuropsychopharmacol 2002; 26:643-52

8. Miller A. Mechanism of cytokine induced behavioral changes. Brain Behav Immun 2009; 23(2):149-58

9. Dantzer R., O'Connor J.C., Freund G.G., Johnson R.W., Kelley K.W. From inflammation to sickness and depression: when the immune system subjugates the brain. Nat Rev Neurosci 2008; 9:46-56.

10. Willeit M., Sitte H.H., Thierry N., et al. Enhanced Serotonin Transporter Function during Depression in Seasonal Affective Disorder. Neuropsychopharmacol 2008; 33(7):1503-13.

\section{CONCLUSIONS}

In our study, urinary dopamine was significantly lower during interferon alpha2b administration in patients with melanoma. The authors calculated a relation defined by a high, statistically significant odds ratio between dopamine status and depression. This correlation suggests that dopamine decrease might play an important role in neuropsychiatric disorders associated with interferon treatment. These results could induce prophylactic pharmacological interventions in dopamine metabolism for reducing neuropsychiatric adverse reactions associated with interferon treatment.

Conflict of interest: None declared.

11. Kitagami T., Yamada K., Miura H., Hashimoto R., Nabeshima T., Ohta T. Mechanism of systemically injected interferon-alpha impeding monoamine biosynthesis in rats: role of nitric oxide as a signal crossing the blood-brain barrier. Brain Res 2003; 978:104-14

12. Moron J.A., Zakharova I., Ferrer J.V., et al. Mitogen-activated protein kinase regulates dopamine transporter surface expression and dopamine transport capacity. J Neurosci 2003; 23:8480-88

13. Dunlop B.W., Nemeroff C.B. The role of dopamine in the pathophysiology of depression. Arch Gen Psychiatry 2007; 64:327-37

14. Miller A., Maletic V., Raison C.L., Inflammation and Its Discontents: The Role of Cytokines in the Pathophysiology of Major Depression, Biol Psychiatry 2009; 65(9):732-41

15. Marc D., Ailts J., Campeau D., Bull M., Olson K. Neurotransmitters excreted in the urine as biomarker of nervous system activity:Validity and clinical applicability. Neurosci Biobehav Rev 2010; doi:10.1016/j. neubiorev.2010.07.007

16. Hasler G. Pathophysiology of depression: do we have any solid evidence of interest to clinicians?. World Psychiatry 2010; 3:155-61.

17. Goldberg J.F., Burdick K.E., Endick C.J. Preliminary randomized, double-blind, placebo- controlled trial of pramipexole added to mood stabilizers for treatment-resistant bipolar depression. Am J Psychiatry 2004: 161:564-66.

18. Lambert G., Johansson M., Agren H., Friberg P. Reduced brain norepinephrine and dopamine release in treatment-refractory depressive illness: evidence in support of the catecholamine hypothesis of mood disorders. Arch Gen Psychiatry 2000; 57:787-93

19. Meyer J.H., Krüger S., Wilson A.A. et al. Lower Dopamine transporter binding potential in striatum during depression. Neuroreport 2001; 12:4121-25.

20. Fontana R.J. Neuropsychiatric toxicity of antiviral treatment in chronic hepatitis C. Dig Dis 2000; 18:107-16 